\title{
Succinate receptor 1 inhibits mitochondrial respiration in cancer cells addicted to glutamine
}

\author{
Philipp Rabe ${ }^{1}$, Aenne-Dorothea Liebing ${ }^{1}$, Petra Krumbholz ${ }^{1}$, Robert Kraft ${ }^{2}$, Claudia Stäubert ${ }^{1 *}$ \\ ${ }^{1}$ Rudolf Schönheimer Institute of Biochemistry, Faculty of Medicine, Leipzig University, \\ Johannisallee 30, 04103 Leipzig, Germany \\ 2 Carl Ludwig Institute for Physiology, Faculty of Medicine, Leipzig University, \\ Liebigstraße 27, 04103 Leipzig, Germany
}

\footnotetext{
*To whom correspondence and requests for materials should be addressed:
}

Claudia Stäubert, Rudolf Schönheimer Institute of Biochemistry, Faculty of Medicine, Leipzig University, Johannisallee 30, 04103 Leipzig, Germany, Tel.: +49-341-9722-157, Fax: +49-3419722-159, Email: claudia.staeubert@medizin.uni-leipzig.de

\begin{abstract}
Cancer cells display metabolic alterations to meet the bioenergetic demands for their high proliferation rates. Succinate is a central metabolite of the tricarboxylic acid (TCA) cycle, but was also shown to act as an oncometabolite and to specifically activate the succinate receptor 1 (SUCNR1), which is expressed in several types of cancer. However, functional studies focusing on the connection between SUCNR1 and cancer cell metabolism are still lacking. In the present study, we analyzed the role of SUCNR1 for cancer cell metabolism and survival applying different signal transduction, metabolic and imaging analyses. We chose a gastric, a lung and a pancreatic cancer cell line for which our data revealed functional expression of SUCNR1. Further, presence of glutamine (Gln) caused high respiratory rates and elevated expression of SUCNR1. Knockdown of SUCNR1 resulted in a significant increase of mitochondrial respiration and superoxide production accompanied by an increase in TCA cycle throughput and a reduction of cancer cell survival in the analyzed cancer cell lines. Combination of SUCNR1 knockdown and treatment with the chemotherapeutics cisplatin and gemcitabine further increased cancer cell death. In summary, our data implicates that SUCNR1 is crucial for Gln-addicted cancer cells by limiting TCA cycle throughput, mitochondrial respiration and the production of reactive oxygen species, highlighting its potential as a pharmacological target for cancer treatment.
\end{abstract}

Keywords

SUCNR1 / GPR91 / metabolite-sensing GPCR / cancer metabolism / glutaminolysis 


\section{Abbreviations}

AA, Antimycin A; ACTB, beta-actin; ATP, adenosine triphosphate; cAMP, cyclic adenosine monophosphate; CoQ, Coenzyme Q, Ubiquinone; Cyt c, cytochrome c; DMR, dynamic mass redistribution; ECAR, extracellular acidification rate; ELISA, enzyme-linked immunosorbent assay; ERK 1/2, extracellular-signal regulated kinases 1/2; ETC, electron transport chain; FCCP, Carbonyl cyanide 4-(trifluoromethoxy)phenylhydrazone; $\mathrm{FADH}_{2}$, flavin adenine dinucleotide; Glc, Glucose; Gln, Glutamine; GPCR, G protein-coupled receptor; HBSS, Hank's Balanced Salt Solution; HIF-1 $\alpha$, hypoxia inducible factor- $1 \alpha$; Hoechst, Hoechst 33342; MMA, methylmalonic acid; NADH, nicotinamide adenine dinucleotide; NMOC, non-mitochondrial oxygen consumption; OA, Oligomycin A; OCR, oxygen consumption rate; OD, optical density; PI, propidium iodide; PI3K, phosphoinositide 3-kinase; POD, peroxidase; PTX, pertussis toxin; ROS, reactive oxygen species; Rot, Rotenone; RPS18, 40S ribosomal protein 18; RT-qPCR, real time-quantitative polymerase chain reaction; $\mathrm{SDH}$, succinate dehydrogenase; TCA, tricarboxylic acid; UBO, UBO-QIC; vh, vehicle

\section{Introduction}

A hallmark of cancer is the dysregulation of cellular metabolism to meet the bioenergetic demands associated with its high proliferation rates [1,2]. Besides aerobic glycolysis, first described by Otto Warburg, it has become increasingly clear that maintenance of mitochondrial function is equally essential for tumor growth as is the availability of glucose [3,4]. Moreover, many cancers are metabolically addicted to glutamine, which, through its conversion to $\alpha$-ketoglutarate, directly fuels the tricarboxylic acid (TCA) cycle anaplerotically, a process called glutaminolysis $[5,6]$. The TCA cycle is crucial for the biosynthesis of lipids, amino acids, and nucleotides but also the main source of energy for cells, by generation of NADH and $\mathrm{FADH}_{2}$, which are further converted to ATP during aerobic mitochondrial respiration [7,8]. A very central metabolite of the TCA cycle is succinate, metabolized by the succinate dehydrogenase (SDH), an enzyme coupling the electron transport chain (ETC) to the oxidation of succinate and $\mathrm{FADH}_{2}$ to fumarate. Further, it has become increasingly clear that succinate is not only a central metabolite, but also acts as an inflammatory signal, carcinogenic initiator and reaches high concentration in the tumor microenvironment [9]. Mutations in SDH cause succinate accumulation in certain cancers like gastric [9], thyroid [10] and lung cancer [11], which is associated with the induction of a pseudo-hypoxic state. Mechanistically this is realized by the succinate-mediated inhibition of prolyl hydroxylases that, under normoxic conditions, hydroxylate the hypoxia inducible factor- $1 \alpha$ (HIF-1 $\alpha$ ), thus targeting it for degradation $[9,12]$. The stabilization of HIF-1 $\alpha$ is important for the switch from mitochondrial oxidative phosphorylation to aerobic glycolysis observed in many cancer cells [13]. Apart from its relevance in cellular metabolism, succinate acts as a specific agonist at the succinate receptor 1 (SUCNR1/GPR91), which is a G protein-coupled receptor (GPCR), causing subsequent activation of $\mathrm{G} \alpha_{\mathrm{i}}$ and $\mathrm{G} \alpha_{\mathrm{q}}$ protein signaling [14]. In addition, activation of SUCNR1 has been demonstrated to induce phosphorylation of Akt in platelets [15] and ERK 1/2 in kidney cells [16].

Physiologically, SUCNR1 is involved in diverse processes including renin-induced hypertension, immune responses, inflammation and inhibition of lipolysis (reviewed in [14]). However, little is known about the expression and relevance of SUCNR1 in cancer [17]. Recently, it has been reported that SUCNR1 is abundantly expressed in lung cancer and promotes cancer metastasis via PI3K/Akt signaling [11]. In ovarian cancer, SUCNR1 was found to be upregulated and, with its high expression, worse progression-free survival was predicted [18]. Similarly, in paragangliomas and pheochromocytomas with SDH mutations, a growth advantage via SUCNR1 was revealed [19]. These findings point towards a pro-cancerous role of SUCNR1, which could serve as potential target in cancer therapy, by blocking its function with specific antagonists. Yet, more functional studies are needed to understand the role of 
SUCNR1 and its connection to metabolism in cancer [17].

Here, we chose cancer cell lines originating from various tissues with high SUCNR1 expression to investigate its role for cancer cell proliferation and regulation of (mitochondrial) metabolism and respiration.

We confirmed functional expression of SUCNR1 by signal transduction analyses in the human gastric adenocarcinoma cell line MKN-45, the lung cancer cell line NCI-H460 and the pancreatic adenocarcinoma cell line PSN-1. Knockdown of SUCNR1 caused a decrease in cancer cell survival in all analyzed cancer cell lines. Further, attempting to link SUCNR1 to the regulation of central cellular metabolism revealed that presence of glutamine causes an increase in SUCNR1 expression, while increased mitochondrial respiration and generation of reactive oxygen species (ROS) accompany its knockdown. Our data suggests that SUCNR1 activation by increased levels of succinate inhibits mitochondrial respiration, thereby potentially reducing the production of extensive ROS. In conclusion, this indicates that SUCNR1 constitutes a negative feedback mechanism, which controls excess mitochondrial activity. At last, we demonstrate that knockdown of SUCNR1 in combination with the well-established chemotherapeutic agents cisplatin and gemcitabine causes increased cancer cell death suggesting that SUCNR1 might be a promising new pharmacological target for cancer therapy.

\section{2. Materials and Methods}

\subsection{Chemicals and antibodies}

All compounds and inhibitors were purchased from Sigma-Aldrich, Cayman Chemical or Santa Cruz Biotechnology if not stated otherwise. All media, antibiotics, fetal bovine serum (FBS) and antibodies were purchased from Thermo Fisher Scientific. The Hank's Balanced Salt Solution (HBSS) was supplemented with $20 \mathrm{mM}$ HEPES and $\mathrm{pH}$ was adjusted to 7.4.

\subsection{Cell culture and functional assays}

All cell lines were obtained from the American Type Culture Collection or the Leibniz Institute DSMZ (German Collection of Microorganisms and Cell Cultures). MKN-45 gastric adenocarcinoma cells (DSMZ no.: ACC 409) and NCI-H460 lung carcinoma cells (DSMZ no.: ACC 737) were cultured in Roswell Park Memorial Institute (RPMI) 1640 medium with $4.5 \mathrm{~g} / 1$ D-glucose. PSN-1 pancreatic adenocarcinoma cells (ATCC CRM-CRL-3211) were grown in RPMI 1640 medium. The human embryonic kidney cell line HEK293-T (ATCC CRL-3216) was cultured in Dulbecco's Modified Eagle medium (DMEM). All cells were maintained in a humidified $5 \% \mathrm{CO}_{2}$ incubator at $37{ }^{\circ} \mathrm{C}$ and to all media $10 \%$ fetal bovine serum (FBS), 100 $\mathrm{U} / \mathrm{ml}$ penicillin and $100 \mu \mathrm{g} / \mathrm{ml}$ streptomycin was added. If not stated otherwise, cancer cells were seeded in 96-well plates (MKN-45: 5 × 104 cells/well; NCI-H460: 1 x 10 4 cells/well; PSN1: $1.5 \times 10^{4}$ cells/well) and 12 -well plates $\left(\mathrm{MKN}-45: 5 \times 10^{5}\right.$ cells/well; NCIH460: 1 x $10^{5}$ cells/well; PSN-1: $1.5 \times 10^{5}$ cells/well) for experiments.

\subsubsection{Dynamic mass redistribution (DMR) assay}

130 For the measurement of receptor activation, the label-free dynamic mass redistribution assay

131 (Corning Epic Biosensor Measurements; Corning Life Sciences) was used. Cancer cells were 132 seeded in fibronectin-coated Epic 384-well microplates (MKN-45: 5 x $10^{4}$ cells/well; NCI-H460: 1 x 10 4 cells/well; PSN-1: $1.5 \times 10^{4}$ cells/well) and prior the experiment treated with $3 \mu \mathrm{g} / \mathrm{ml}$ PTX for $12 \mathrm{~h}$ or $300 \mathrm{nM}$ Ubo for $30 \mathrm{~min}$, respectively. Equilibration was performed in HBSS/HEPES for $2 \mathrm{~h}$ followed by stimulation with $200 \mu \mathrm{M}$ epoxysuccinate and 40 min DMR recording.

138 We used the ALPHAScreen cAMP Detection Kit (Perkin Elmer) for measurement of 139 intracellular cyclic adenosine monophosphate (cAMP) levels. Cancer cells were seeded in 140 96-well plates and stimulated (vehicle or $200 \mu \mathrm{M}$ epoxysuccinate) in HBSS with $1 \mathrm{mM} 3-$ 141 isobutyl-1-methylxanthine (IBMX) and $10 \mu \mathrm{M}$ forskolin for $15 \mathrm{~min}$ at $37^{\circ} \mathrm{C}$. After that, the 
stimulation solution was removed and $100 \mu 1$ lysis buffer with $1 \mathrm{mM}$ IBMX was added to each well. $5 \mu 1$ of each lysate was transferred to a 384-well plate, acceptor and donor beads were added and fluorescence was measured following the manufacturer's protocol (Perkin Elmer).

\subsubsection{Alpha SureFire Ultra Multiplex pAkt1/2/3 (Ser473) \& total Akt1 Assay and pERK} 1/2 (Thr202/Tyr204) \& total ERK Assay

To determine the cellular pAkt/total Akt and pERK/total ERK levels the Alpha SureFire Ultra Multiplex pAkt

1/2/3 (Ser473) \& total Akt1 assay and the pERK 1/2 (Thr202/Tyr204) \& Total ERK assay (Perkin Elmer) was used. Cancer cells were seeded in 96-well plates and stimulated with or without $200 \mu \mathrm{M}$ epoxysuccinate in HBSS + Gln for $30 \mathrm{~min}$ at $37{ }^{\circ} \mathrm{C}$. After that, stimulation buffer was removed and $50 \mu \mathrm{l}$ of the supplied lysis buffer was added to each well. $10 \mu 1$ of each lysate was transferred to a 384-well plate, acceptor and donor beads were added and fluorescence was measured following the manufacturer's protocol (Perkin Elmer).

\subsubsection{Calcium Imaging}

Cancer cells were seeded on glass cover slips in 24-well plates and, on the day of the imaging, loaded with $5 \mu \mathrm{M}$ Fura-2 AM (Molecular Probes) in standard solution containing $140 \mathrm{mM}$ $\mathrm{NaCl}, 10 \mathrm{mM}$ HEPES, $5 \mathrm{mM} \mathrm{KCl}, 2 \mathrm{mM} \mathrm{CaCl}_{2}, 1 \mathrm{mM} \mathrm{MgCl}_{2}$, and $10 \mathrm{mM}$ glucose for 30-60 min, respectively. A monochromator-based imaging system and the imaging software TILLvisION 4.0 (T.I.L.L. Photonics) was used for Fura-2 based calcium imaging in single cells. The fluorescence signal (Excitation: $340 \mathrm{~nm}$ and $380 \mathrm{~nm}$ ) was measured with a CCD camera (PCO Imaging) at $2 \mathrm{sec}$ intervals and corrected for background fluorescence. Via bath perfusion, the cancer cells were stimulated with the SUCNR1 agonists succinate $(200 \mu \mathrm{M})$, epoxysuccinate $(200 \mu \mathrm{M})$ and methylmalonic acid (MMA, $400 \mu \mathrm{M})$, which were dissolved in standard solution.

\section{6}

167

168

169

170

171

172

\subsection{5 siRNA transfection}

Cancer cells were transfected with 7.5 pmol siRNA directed against SUCNR1 mRNA (siSUCNR1-I: rArCrUrGrGrUrUrUrCrUrUrCrCrUrCrUrArArGrArArUrUGA, siSUCNR1-II: rCrArUrGrGrArArUrGrCrArArCrUrUrGrCrArArArArArCTG), scrambled siRNA (siNC) or fluorescently labelled siRNA (siTC) (OriGene) using SAINT-sRNA (Synvolux). $11.5 \mu 1$ (96well plate) or $115 \mu \mathrm{l}$ (12-well plate) of the siRNA/SAINT-sRNA mix was added to the medium. Cells transfected with siTC were imaged with the Celigo Image Cytometer (Nexcelom) after $48 \mathrm{~h}$ to detect siRNA transfection efficiency. Cancer cell RNA was prepared for determination of siRNA knockdown efficiency $48 \mathrm{~h}$ after siRNA transfection.

\subsubsection{Determination of SUCNR1 protein knockdown efficiency}

HEK-293T cells were seeded in T-25 cell culture flasks $\left(1.6 \times 10^{6}\right.$ cells/flask $)$ and cotransfected with siSUCNR1-II or siNC, respectively, and plasmid encoding N-terminally HA- and C-terminally FLAG-tagged SUCNR1. Lipofectamine 2000 (Life Technologies, Darmstadt, Germany) with $2 \mu \mathrm{g}$ plasmid and 200 pmol siRNA was used for transient transfection. $24 \mathrm{~h}$ after transfection, cells were harvested and plated in 48 -well plates $\left(1 \times 10^{5}\right.$ cells/well; cell surface expression) or 6 -well plates $\left(5 \times 10^{5}\right.$ cells/well; total receptor expression). SUCNR1 cell surface and total protein expression were measured using an indirect cellular and a sandwich ELISA, respectively. Both assays are described in [20].

\subsection{SUCNR1 mRNA expression analysis and cloning}

For SUCNR1 mRNA expression analysis in 2D and 3D cell culture, cancer cells were cultured in 12-well plates as a 2D monolayer or as 3D spheroids in 96-well ultra-low attachment spheroid microplates (Nexcelom; $5 \times 10^{3}$ cells/well) and RNA was prepared $48 \mathrm{~h}$ after seeding. To analyze the impact of Gln, itaconate, $\mathrm{CoCl}_{2}$ or hypoxia on SUCNR1 expression, cancer cells 
were plated in 12-well plates and, after $48 \mathrm{~h}$, incubated for $4 \mathrm{~h}$ in HBSS, HBSS + Gln, HBSS + $190 \mathrm{Gln}+$ itaconate or HBSS $+\mathrm{Gln}+\mathrm{CoCl}_{2}$, respectively, in a non- $\mathrm{CO}_{2}$ incubator at $37{ }^{\circ} \mathrm{C}$ or in 191 HBSS + Gln in a hypoxic chamber.

\subsubsection{RNA preparation, reverse transcription, quantitative real-time PCR} following the company's protocol and yielded RNA was stored at $-80^{\circ} \mathrm{C}$. The RNA following the company's protocol and yielded RNA was stored at $-80{ }^{\circ} \mathrm{C}$. The RNA concentration was determined with the NanoDrop ND-1000 and an additional DNase I (NEB) digestion was performed at $37{ }^{\circ} \mathrm{C}$ for $30 \mathrm{~min}$ before reverse transcription to remove any remaining DNA. Afterwards DNase I was inactivated by addition of EDTA $(5 \mathrm{mM})$ and incubation at $75{ }^{\circ} \mathrm{C}$ for 10 min. Finally, the iScript cDNA Synthesis Kit (Bio-Rad) was used to transcribe RNA (500 ng) in cDNA. The cDNA solution was diluted with nuclease-free water to a final volume of $70 \mu$ l.

Quantitative real-time PCR (qPCR) was performed with $1 \mu \mathrm{l}$ cDNA, $1 \mu 1$ primer mix with sense- and antisense-primer $(400 \mathrm{nM}$ of each primer, SUCNR1-S: CACATCTGGAGTGAGCCTTGT, SUCNR1-AS: GGGCCTCATTAACCCTCCTG ), $5 \mu 1$ Luna Universal qPCR Master Mix (NEB) and $5 \mu$ l nuclease-free water on the CFX Connect Real-Time PCR Detection System (Bio-Rad). qPCR was initiated with the activation of the DNA polymerase at $95{ }^{\circ} \mathrm{C}$ for 2 min followed by 40 cycles of denaturation at $95^{\circ} \mathrm{C}$ for $15 \mathrm{sec}$ and primer annealing and elongation at $60^{\circ} \mathrm{C}$ for $30 \mathrm{sec}$. Fluorescence was measured at the end of each annealing/elongation step. Lastly, melt curves were recorded from $55^{\circ} \mathrm{C}$ to $95{ }^{\circ} \mathrm{C}(0.5$ ${ }^{\circ} \mathrm{C}$ increment, $5 \mathrm{sec}$ per step) and showed one single peak for each primer pair. Primers were designed with Primer-Blast (http://www.ncbi.nlm.nih.gov/tools/primer-blast/) and purchased from Microsynth Seqlab. For calculation of $\Delta$ ct values, the mRNA expression of the reference genes $A C T B$ (ACTB-S: ACAATGTGGCCGAGGACTTT, ACTB-AS: TGGGGTGGCTTTTAGGATGG) and RPS18 (RPS18-S: GATGGGCGGCGGAAAATAG, RPS18-AS: GTCTGCTTTCCTCAACACCAC) was also determined.

\subsubsection{Amplification and cloning of SUCNR1}

Primer pairs, positioned in the 5'- (GGCAGAGTTCCTGTCAAGGG) and 3'-UTR (CTTGTCGTCCCATTCTTTTCACA) were used to amplify SUCNR1 from cDNA of cancer cell lines. PCR reactions were performed with Q5 High-Fidelity DNA Polymerase following the manufacturer's instructions. SUCNR1 was epitope-tagged with an N-terminal hemagglutinin (HA) epitope and a C-terminal FLAG-tag by a PCR-based overlapping fragments approach and inserted into the mammalian expression vector pcDps. Identity and correctness of the construct was confirmed by sequencing (SeqLab).

\subsection{Image-based analyses}

\subsubsection{Cancer cell growth and survival analyses}

Cancer cells were cultured as 2D monolayers in black 96-well plates with clear bottom (Greiner No. 655090) or as 3D spheroids in ultra-low attachment spheroid microplates (Nexcelom; $5 \mathrm{x}$ $10^{3}$ cells/well), transfected with SUCNR1-specific siRNA (siSUCNR1-I, siSUCNR1-II) or siNC and confluence or spheroid volume was measured every $24 \mathrm{~h}$ with the Celigo Image Cytometer (Nexcelom). Cells were stained $24 \mathrm{~h}$ after siRNA transfection to determine total (Hoechst 33342, Hoechst, $1 \mu \mathrm{g} / \mathrm{ml}$, Sigma-Aldrich) and apoptotic cells (Annexin V-AF488, AAT Bioquest) or $72 \mathrm{~h}$ after siRNA transfection to determine total (Hoechst, $1 \mu \mathrm{g} / \mathrm{ml}$ ) and dead cells (propidium iodide, PI, $1 \mu \mathrm{g} / \mathrm{ml}$, Thermo Fisher Scientific) using the Celigo Image Cytometer.

\subsection{2 siSUCNR1 in combination with cisplatin or gemcitabine survival analyses}

Cancer cells were seeded in black 96-well plates with clear bottom (Greiner No. 655090) and transfected with SUCNR1-specific siRNA (siSUCNR1-I, siSUCNR1-II) or siNC $24 \mathrm{~h}$ after seeding. Cisplatin or gemcitabine $(10 \mu \mathrm{M})$, respectively, was added to the medium $24 \mathrm{~h}$ after 
siRNA transfection. For the cisplatin dose-response curves various concentrations of cisplatin were added to the medium $24 \mathrm{~h}$ after siRNA transfection. Cells were stained $48 \mathrm{~h}$ later to determine total (Hoechst, $1 \mu \mathrm{g} / \mathrm{ml}$ ) and dead cells (PI, $1 \mu \mathrm{g} / \mathrm{ml}$ ) using the Celigo Image Cytometer.

\subsubsection{CQ1 Confocal Imaging} $48 \mathrm{~h}$ after seeding incubated in HBSS $+\mathrm{Cln}$ for $4 \mathrm{~h}$ at $37^{\circ} \mathrm{C}$ in a $48 \mathrm{~h}$ after seeding incubated in HBSS + Gln for $4 \mathrm{~h}$ at $37{ }^{\circ} \mathrm{C}$ in a non- $\mathrm{CO}_{2}$ incubator. After the incubation, cells were fixed in PBS with $4 \%$ formaldehyde and permeabilized with $0.5 \%$ Triton-X in PBS. Cells were washed twice with PBS after each step. After that, $100 \mu 1$ medium with $10 \%$ FBS was added to each well and cells were incubated for $30 \mathrm{~min}$ at $37^{\circ} \mathrm{C}$ to block non-specific binding sites. For HIF-1 $\alpha$ staining, cells were incubated with primary mouse antiHIF-1 $\alpha$ antibody (1:100 in medium) for $2 \mathrm{~h}$ at $37^{\circ} \mathrm{C}$. After washing with PBS, secondary AlexaFluor 488 goat anti-mouse antibody was added (1:500 in medium) and cells were incubated for $1 \mathrm{~h}$ at $37^{\circ} \mathrm{C}$. Following the antibody staining, cells were washed with PBS and nuclei were stained with Hoechst (Thermo Fisher Scientific) for $30 \mathrm{~min}$ at $37^{\circ} \mathrm{C}$. Confocal imaging was performed using the CQ1 Confocal Imaging Cytometer (Yokogawa, Cenibra).

\subsubsection{Measurement of mitochondrial superoxide}

The production of mitochondrial superoxide was measured using the MitoSOX Red Mitochondrial Superoxide Indicator (Thermo Fisher Scientific). Cancer cells were seeded in black 96-well plates with clear bottom (Greiner No. 655090) and transfected with siSUCNR1 or siNC $24 \mathrm{~h}$ after seeding. $48 \mathrm{~h}$ after siRNA transfection, cells were incubated in HBSS + Gln for $2 \mathrm{~h}$ and subsequently stained with Hoechst $(1 \mu \mathrm{g} / \mathrm{ml})$ and MitoSOX $(5 \mu \mathrm{M})$ for $10 \mathrm{~min}$ at $37^{\circ} \mathrm{C}$. Lastly, staining solution was removed, $100 \mu \mathrm{HBSS}+$ Gln was added to each well and fluorescence was measured using the Celigo Image Cytometer.

\subsection{Seahorse XF Cell Mito Stress Test}

Metabolic analyses were performed using the Seahorse XF Cell Mito Stress Test Kit and the XFe96 Analyzer (Seahorse Bioscience). Cancer cells were seeded in Poly-L-Lysine coated XF96 cell culture microplates (MKN-45: $2 \times 10^{4}$ cells/well; NCI-H460: 1 × $10^{4}$ cells/well; PSN-1: $3 \times 10^{4}$ cells/well) and incubated at $37{ }^{\circ} \mathrm{C}$ and $5 \% \mathrm{CO}_{2} .24 \mathrm{~h}$ before the assay, cancer cells were transfected with siNC to test the impact of different supplements (Glc, Gln, pyruvate) on cancer cell metabolism. For the analysis of SDH inhibition, cells were treated with $10 \mathrm{mM}$ itaconate $4 \mathrm{~h}$ prior to the assay. To analyze the role of SUCNR1 in cancer cell metabolism, cells were transfected with siSUCNR1 and siNC $24 \mathrm{~h}$ before the assay. On the day of the assay, growth medium with siRNA or itaconate was removed and cells were washed twice with XF RPMI medium $\left(37^{\circ} \mathrm{C}\right.$; $\left.\mathrm{pH} 7.4\right)$ supplemented with either Glc $(10 \mathrm{mM})$, Gln $(2 \mathrm{mM})$, Glc and sodium pyruvate $(10 \mathrm{mM}, 1 \mathrm{mM})$ or Gln and sodium pyruvate $(2 \mathrm{mM}, 1 \mathrm{mM})$. Finally, $180 \mu 1$ of the assay medium was added to the cells and the microplates were placed in a $37^{\circ} \mathrm{C}$ incubator without $\mathrm{CO}_{2}$ for $1 \mathrm{~h}$.

The respiratory chain inhibitors Oligomycin A (OA), Carbonyl cyanide-4 (trifluoromethoxy) phenylhydrazone (FCCP) and Rotenone/Antimycin A (Rot/AA) that are used for the determination of different mitochondrial key parameters were solved and loaded into the ports of the sensor cartridge following the manufacturer's protocol (Seahorse Bioscience). The ETC consists of complex I (inhibited by Rotenone), complex II/succinate dehydrogenase, complex III (inhibited by Antimycin A) and complex IV. The electrochemical proton gradient generated by the electron transport is used for ATP generation by complex V/ATP synthase (inhibited by Oligomycin A). FCCP is a chemical uncoupler, which disrupts the proton gradient and mitochondrial membrane potential allowing maximal oxygen consumption. The final well concentrations in the experiments were: $1.5 \mu \mathrm{M}$ OA, $2 \mu \mathrm{M}$ FCCP and $0.5 \mu \mathrm{M}$ Rot/AA. After 15 to 30 min calibration of the cartridge in the XFe96 Analyzer, the utility plate was replaced 
with the cell culture microplate and the assay was started. Oxygen consumption rate (OCR) and extracellular acidification rate (ECAR) were determined every 6 minutes for 18 minutes under basal conditions as well as after consecutive OA, FCCP and Rot/AA injection. After the assay, cells were stained with Hoechst $(1 \mu \mathrm{g} / \mathrm{ml})$ and the cell number in each well was determined with the Celigo Image Cytometer. Finally, OCR and ECAR were normalized to 10000 cells.

\subsection{Data analyses}

All data were statistically analyzed and visualized using GraphPad Prism 7 for Windows (GraphPad Software, San Diego California USA, www.graphpad.com). Detailed information about statistical analysis are included in each figure legend.

\section{Results}

\subsection{SUCNR1 is functionally expressed in 2D and 3D cultured cancer cell lines}

SUCNR1 is expressed in different types of cancer like lung [11] and ovarian [18] cancer. Database mining revealed high expression of SUCNR1 in several cell lines. Here, we chose cancer cell lines originating from different tissues and compared the SUCNR1 mRNA expression between 2D monolayer cell cultures and 3D spheroids using RT-qPCR. The lung carcinoma cells NCI-H460 formed densely packed 3D spheroids, whereas spheroids of the gastric adenocarcinoma cells MKN-45 and the pancreatic adenocarcinoma cells PSN-1 were relatively loose when the same number of cells $\left(5 \times 10^{3}\right.$ cells/well) was seeded into the plates (Figure 1A). We confirmed high SUCNR1 mRNA expression in all three cancer cell lines (Figure 1B). Only for spheroids of NCI-H460 cells, we observed a lower $\Delta c_{t}$ value, reflecting an increased SUCNR1 expression compared with 2D cultures (Figure 1B). Next, we analyzed functional expression of SUCNR1 and downstream signaling pathways in the cancer cell lines. For this purpose, we used epoxysuccinate, a SUCNR1 agonist with 10- to 20-fold higher potency than succinate and which is not metabolized in the TCA cycle [21]. This ensured that any detected signaling is specific for SUCNR1 and not caused by potential metabolic effects of succinate. Label-free dynamic mass redistribution (DMR) measurements were used to detect signal transduction upon SUCNR1 activation close to the cell surface independently of certain signaling pathways. Utilization of inhibitors selectively blocking specific signal transduction components enables distinction of the involved pathways. Since it is known that SUCNR1 is a $\mathrm{G} \alpha_{\mathrm{i}} / \mathrm{G} \alpha_{\mathrm{q}}$ coupled receptor, we used the inhibitors pertussis toxin (PTX), blocking $\mathrm{G} \alpha_{\mathrm{i}}$ signaling, and UBO-QIC (UBO) [22], blocking $\mathrm{G} \alpha_{\mathrm{q}}$ signaling, to see if these pathways contribute to the SUCNR1-induced DMR signal. All three cell lines were stimulated with $200 \mu \mathrm{M}$ epoxysuccinate and DMR was recorded for 40 minutes. However, only the NCI-H460 cells showed a measurable DMR response that was strongly inhibited after $16 \mathrm{~h}$ incubation with PTX indicating a high contribution of the $\mathrm{G} \alpha_{i}$ signaling pathway to SUCNR1 signaling (Figure 1C). In contrast, UBO did not affect the DMR signal caused by stimulation with epoxysuccinate. Since no measureable DMR response was detected for MKN-45 and PSN-1 we performed cAMP inhibition assays in presence of $10 \mu \mathrm{M}$ forskolin to validate functional presence of SUCNR1 in these two cancer cell lines. G $\alpha_{\mathrm{i}}$-dependent activation upon SUCNR1 stimulation caused a decrease in intracellular cAMP levels, which was sensitive to PTX in both MKN-45 and PSN-1 (Figure 1D). G $\alpha_{\mathrm{q}}$ signaling did not influence cAMP levels as tested by application of the specific inhibitor UBO. However, in MKN-45 SUCNR1 signaling was sensitive to Gallein indicating involvement of G $\beta \gamma$ signaling (Figure 1D). In summary, we observed $\mathrm{G} \alpha_{\mathrm{i}}$ dependent SUCNR1 signal transduction in all three cancer cell lines.

To further test a potential involvement of $\mathrm{G}_{\mathrm{q}}$ signaling, we applied $\mathrm{Ca}^{2+}$ imaging experiments. In accordance with the missing effect of UBO on SUCNR1 signaling in the DMR measurements (Figure $1 \mathrm{C}$ ), $\mathrm{Ca}^{2+}$ signals were absent in the analyzed cancer cell lines upon stimulation with epoxysuccinate (Figure S1A). Akt [15] and ERK 1/2 [16] signaling has been reported for SUCNR1. Here, we confirm Akt signaling in the three tested cancer cell lines (Figure 1E) and 
ERK 1/2 activation in MKN-45 and NCI-H460 cells (Figure 1F) upon receptor activation with epoxysuccinate.
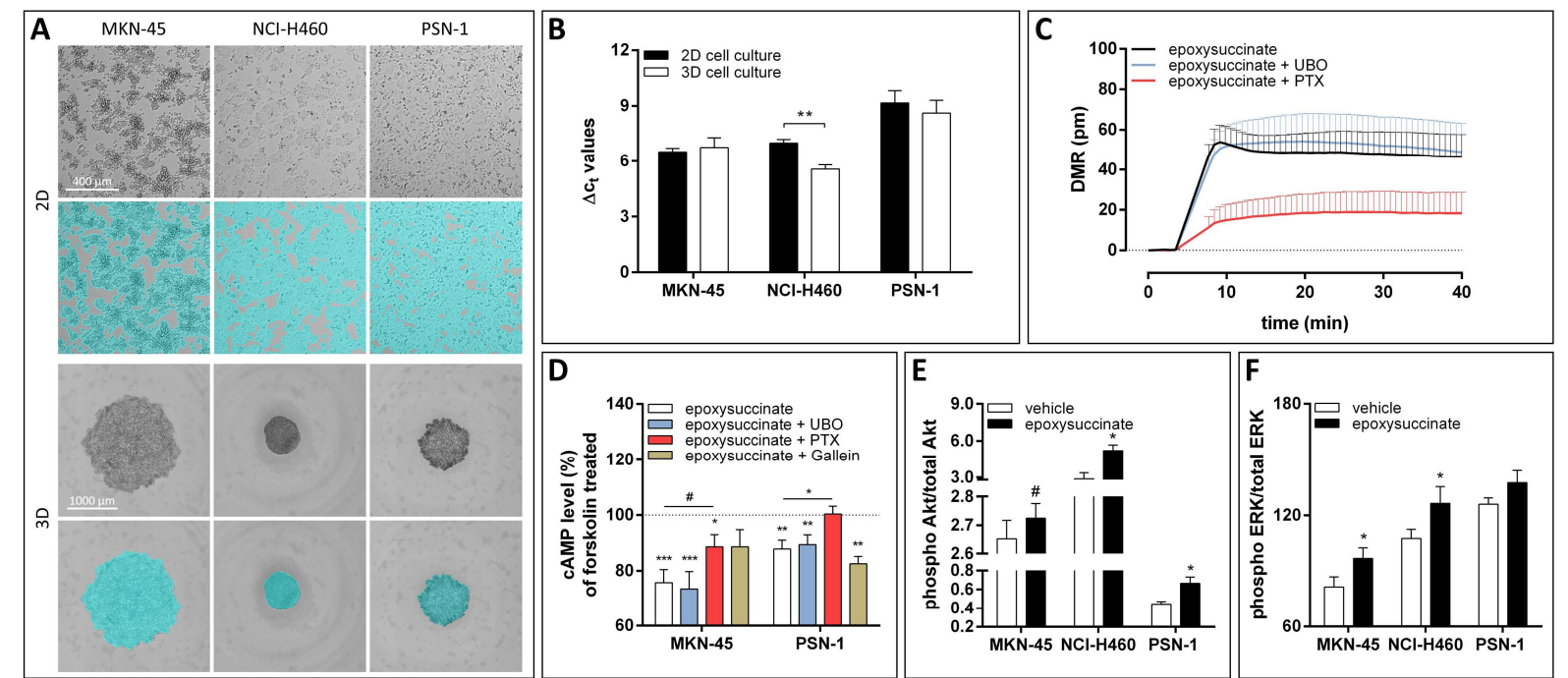

Figure 1: SUCNR1 is abundantly expressed in cancer cell lines and signals via Go, Akt and ERK.

A, B: Cell lines from gastric (MKN-45), lung (NCI-H460) and pancreatic (PSN-1) cancer were cultured in 2D and $3 \mathrm{D}$ cell culture $(\mathrm{A})$ and SUCNR1 mRNA expression $(\mathrm{B})$ was determined. $\Delta \mathrm{c}_{\mathrm{t}}$ values were calculated based on the reference gene expression of $A C T B\left(\mathrm{c}_{\mathrm{t}} \approx 17.5\right)$ and $\operatorname{RPS18}\left(\mathrm{c}_{\mathrm{t}} \approx 20.5\right)$. C: We determined the dynamic mass redistribution (DMR) signal in pm of NCI-H460 cells upon stimulation with $200 \mu \mathrm{M}$ epoxysuccinate in absence or presence of pertussis toxin (PTX) or UBO-QIC (UBO). D: cAMP levels upon stimulation with $200 \mu \mathrm{M}$ epoxysuccinate and $10 \mu \mathrm{M}$ forskolin were measured in absence and presence of PTX, UBO or Gallein, respectively. cAMP level is shown as \% of cells treated with $10 \mu \mathrm{M}$ forskolin. E, F: Phosphorylation of Akt (Ser473) (E) and ERK (Thr202/Tyr204) (F) induced by stimulation with epoxysuccinate $(200 \mu \mathrm{M})$ is shown as ratio of phosphorylated Akt over total Akt (E) or phosphorylated ERK over total ERK (F). Data is shown as mean \pm SEM of $n \geq 3$ independent experiments, each carried out in triplicates. Statistical analyses were performed using an unpaired $(\mathrm{B}, \mathrm{D})$ or a paired t-test $(\mathrm{E}, \mathrm{F}) .{ }^{*} \mathrm{P} \leq 0.1 ; * \mathrm{P} \leq 0.05 ; * * \mathrm{P} \leq 0.01 ; * * * \mathrm{P} \leq 0.001$.

3.2 Presence of glutamine is associated with an increase in SUCNR1 expression and mitochondrial respiration in MKN-45, NCI-H460 and PSN-1 cells

We next asked whether SUCNR1 mRNA expression is linked to certain metabolic demands. We incubated MKN-45, NCI-H460 and PSN-1 cells for $4 \mathrm{~h}$ at $37^{\circ} \mathrm{C}$ in HBSS with glucose (Glc) or HBSS with Glc and glutamine (Gln), respectively and performed RT-qPCR. Compared to HBSS with Glc only, we found significantly elevated SUCNR1 mRNA levels upon addition of Gln (Figure 2A). To further validate the connection between SUCNR1 expression and cellular metabolism, we performed Mito Stress Test Assays with the Seahorse XFe96 Analyzer, which measures oxygen consumption rate (OCR) and extracellular acidification rate (ECAR) in absence and presence of different respiratory chain inhibitors (Figure 2B). These measurements enable conclusions about different metabolic parameters (Figure 2B). First, we determined the effect of the availability of different energy substrates (Glc alone, Glc and pyruvate, Gln alone, Gln and pyruvate) in scrambled negative control siRNA (siNC) transfected MKN-45, NCI-H460 and PSN-1 cells (Figure 2C-2E). With Gln as the only supplement in the medium, all three cancer cell lines showed a highly increased basal and maximal respiration when compared to Glc (Figure 2C) reflecting that Gln is likely used to fuel the tricarboxylic acid (TCA) cycle. Thus, a higher OCR (Figure 2C) reflects an increased metabolic throughput. Furthermore, oxygen consumption coupled to ATP production and non-mitochondrial oxygen consumption (NMOC) is increased in presence of Gln alone (Figure 2D). In contrast to Gln, we observed in presence of Glc alone a significantly increased ECAR (Figure 2E). This suggests that Glc, after its conversion to pyruvate, is mainly metabolized to lactate and, together with protons, exported out of the cell. Next, we tested whether addition of pyruvate, which can be 
converted to acetyl-CoA and enter the TCA cycle further fuels OCR in presence of either Glc or Gln alone (Figure 2C). We found that addition of extracellular pyruvate caused an increased basal and maximal respiration in MKN-45 and NCI-H460 (Figure 2C) cells when added to Glc or Gln. While addition of pyruvate caused a decrease in ECAR in the presence of Glc, the opposite was true for Gln in NCI-H460 cells (Figure 2E). This implicates that extracellular pyruvate induces a metabolic shift towards a more aerobic phenotype in presence of Glc. Pyruvate availability in addition to Gln further fuels the TCA cycle causing, besides higher

OCR, an elevated ECAR due to bicarbonate and proton production during respiration [23,24]. Further, pyruvate caused an increase of NMOC as compared to Glc or Gln alone in MKN-45 cells (Figure 2D). This was only true for Gln in NCI-H460 cells whereas no difference was observed in PSN-1 cells (Figure 2D).

In summary, the three SUCNR1 expressing cancer cell lines strongly depend on Gln (Figure 2F). However, MKN-45, NCI-H460 and PSN-1 cells also exhibited differences in their metabolic features dependent on substrate availability.

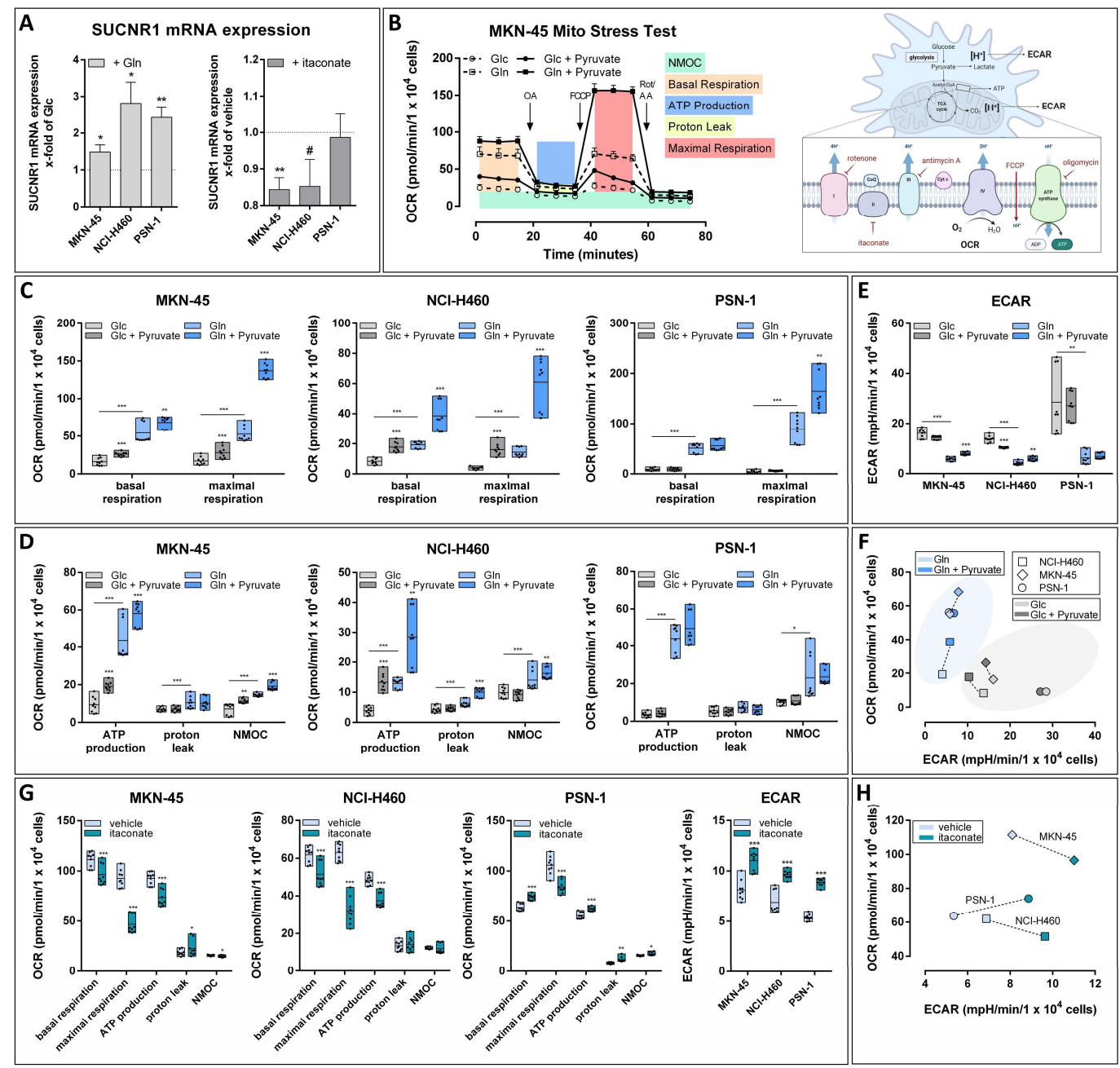

Figure 2: Glutamine (GIn) causes an increase in SUCNR1 mRNA expression and is a crucial energy substrate for the cancer cell lines MKN-45, NCI-H460 and PSN-1.

A: SUCNR1 mRNA levels in cancer cell lines were measured by RT-qPCR after $4 \mathrm{~h}$ incubation in presence of glucose (Glc) or Glc with glutamine (Gln) or upon treatment with $10 \mathrm{mM}$ succinate dehydrogenase (SDH) inhibitor itaconate. Data is shown as mean \pm SEM of $n=4$ independent experiments. B: Oxygen consumption rate (OCR) and extracellular acidification rate (ECAR) of cancer cells in the presence of different supplements were measured in Mito Stress Test Assays using the Seahorse XFe96 Analyzer. By the addition of specific inhibitors of the ETC, different parameters of mitochondrial respiration were determined. ECAR is influenced by the production of lactate but also by $\mathrm{CO}_{2}$, hydrated to $\mathrm{H}_{2} \mathrm{CO}_{3}$, originating from respiration. C, D, E: Gln caused increased respirational parameters and non-mitochondrial oxygen consumption (NMOC) but decreased ECAR compared to 
Glc in all cancer cell lines. The combination of Gln and pyruvate further increased mitochondrial oxygen consumption, NMOC and ECAR in MKN-45 and NCI-H460 cells but not PSN-1. F: Mitochondrial respiration of cancer cells is dependent on glutamine (Gln). With Gln, high mitochondrial oxygen consumption was observed, causing a shift to a more aerobic phenotype. G: Mito stress test assays were performed in RPMI medium + Gln, w/o Glc in absence and presence of $10 \mathrm{mM}$ itaconate causing reduced maximal respiration and increased ECAR in all cancer cell lines. H: Itaconate inhibited SDH dependent mitochondrial respiration affecting oxygen consumption in cancer cells. C, D, E, G: Data is shown as floating bars ( $\min$ to max) of three time points of three independent experiments, each carried out in 6 technical replicates. Statistical analyses were performed using an (A) unpaired t-test, a $(\mathrm{C}, \mathrm{D}, \mathrm{E})$ repeated measures One-Way ANOVA or a $(\mathrm{G})$ paired $\mathrm{t}$-test. ${ }^{\#} \mathrm{P} \leq 0.1 ;{ }^{*} \mathrm{P} \leq 0.05$; $* * \mathrm{P} \leq 0.01 ; * * \mathrm{P} \leq 0.001$.

409

410

411

412

413

414

415

416

417

418

419

420

421

422

423

424

425

426

427

428

429

430

431

432

433

434

435

436

437

438

439

440

441

442

443

444

445

446

447

448

449

\subsection{Inhibition of succinate dehydrogenase with itaconate causes a reduction of mitochondrial respiration}

We aimed to analyze how downregulation of the TCA cycle throughput affects OCR and ECAR in the presence of Gln. Therefore, we used itaconate $(10 \mathrm{mM})$ to selectively inhibit the succinate dehydrogenase (SDH), which converts succinate to fumarate but is also known as complex II of the electron transport chain (ETC) [25]. SDH malfunction is known to be associated with intramitochondrial and intracellular succinate accumulation, but also with an increase of extracellular succinate [26]. Prior to the metabolic analyses, we tested whether inhibition of SDH by itaconate has an impact on SUCNR1 mRNA levels. Treatment with itaconate caused a reduction of SUCNR1 mRNA in MKN-45 and NCI-H460 cells but not PSN-1 cells (Figure 2A). In MKN-45 and NCI-H460 cells, inhibition of SDH caused a decrease in basal respiration, maximal respiration and ATP production, which is in accordance with a decreased TCA cycle metabolic rate (Figure 2G, 2H). In PSN-1 cells, itaconate increased basal respiration and ATP production but decreased maximal respiration (Figure $2 \mathrm{G}, 2 \mathrm{H}$ ). In all three cancer cell lines, ECAR was elevated upon itaconate treatment (Figure 2G, 2H).

\subsection{Knockdown of SUCNR1 increases mitochondrial respiration in cancer cells}

Since SUCNR1 mRNA expression was increased in presence of Gln (Figure 2A), we analyzed whether SUCNR1 is involved in the regulation of mitochondrial respiration when Gln is present. We transfected the cancer cells with two different SUCNR1-specific siRNAs (siSUCNR1-I, siSUCNR1-II) or siNC. A fluorescently labelled siRNA was used to monitor transfection efficiency revealing successful transfection of all cancer cell lines (Figure S1B). Knockdown of SUCNR1 was confirmed at the mRNA level and we found that both siRNAs induced a significant decrease in SUCNR1 mRNA expression by about $33 \%$ (MKN-45), $81 \%$ (NCI-H460) and $75 \%$ (PSN-1), respectively (Figure S1C). To test whether reduced SUCNR1 mRNA levels also lead to decreased protein expression, we co-transfected HEK293-T cells, which do not express SUCNR1 endogenously, with plasmids encoding an HA-tagged SUCNR1 and siSUCNR1-II. POD-coupled anti-HA antibodies were used in surface and total ELISA to confirm a strong reduction of total as well as cell surface protein expression of SUCNR1 (Figure S1D). Since siSUCNR1-I is directed against a SUCNR1 mRNA not located in the open reading frame, it was not included in this control experiment.

Mito Stress Test Assays in RPMI medium with Gln but no Glc as supplement revealed that cancer cells with knockdown of SUCNR1 showed higher basal and/or maximal respiration accompanied by increased ATP-coupled OCR (Figure 3A). SUCNR1 knockdown did not affect proton leak in MKN-45 and PSN-1 but caused an increase in NCI-H460 cells (Figure 3A). Further, NMOC was elevated only in PSN-1 cells with reduced SUCNR1 expression but not in MKN-45 or NCI-H460 cells (Figure 3A). In all three cancer cell lines we found that knockdown of SUCNR1 was associated with an increase in ECAR, which is solely caused by bicarbonate production during respiration because only Gln, but no Glc was present in the medium (Figure 3B). Since presence of pyruvate in addition to Gln caused a further increase in OCR of the cancer cells (Figure 2C), we also analyzed the effect of SUCNR1 knockdown in medium containing both. Addition of pyruvate to Gln containing medium partially reversed the effect 
of SUCNR1 knockdown on mitochondrial respiration (Figure S2A). PSN-1 cells still showed elevated NMOC (Figure S2A) and only MKN-45 cells had an increased ECAR (Figure S2B). Thus, in presence of Gln alone the cancer cells showed higher OCR and ECAR when a knockdown of SUCNR1 was induced. This was mostly reversed upon addition of pyruvate to the medium. Taken together, our results suggest that SUCNR1 serves as negative feedback regulator of mitochondrial respiration and/or TCA cycle throughput in the analyzed cancer cell

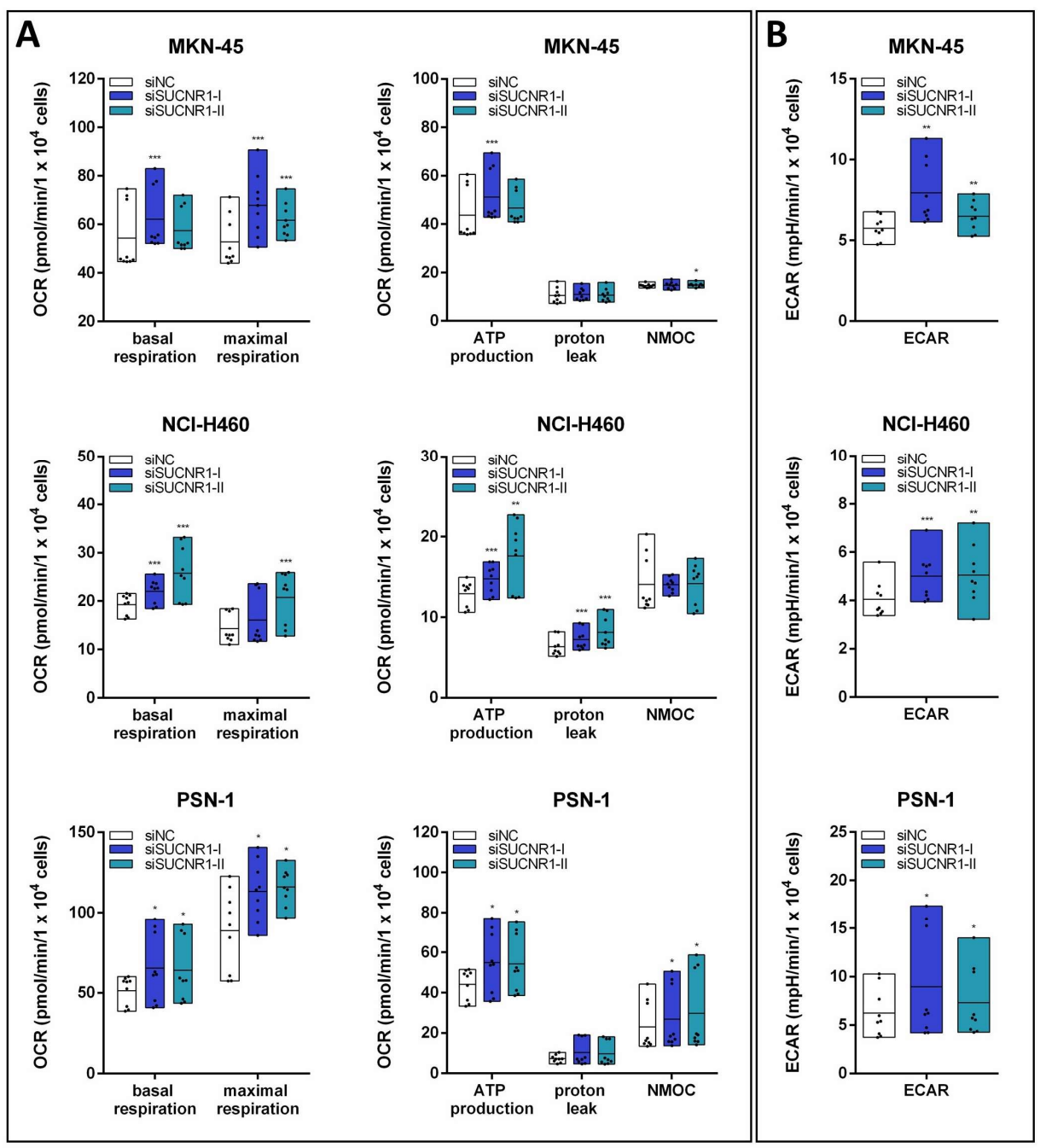
lines.

Figure 3: siRNA mediated knockdown of SUCNR1 is accompanied by increased mitochondrial respiration in cancer cell lines.

$24 \mathrm{~h}$ after cancer cells were transfected with two different siRNAs targeting SUCNR1 or siNC oxygen consumption rate (OCR) and extracellular acidification rate (ECAR) were measured in RPMI medium containing only Gln. Knockdown of SUCNR1 caused an increase in A: basal and maximal respiration, oxygen consumption coupled to ATP production and B: ECAR in all three cancer cell lines. Data is shown as floating bars (min to max) of three time points of three independent experiments, each carried out in 6 technical replicates. Statistical analyses were performed using a repeated measures One-Way ANOVA. * $\mathrm{P} \leq 0.05 ;{ }^{* *} \mathrm{P} \leq 0.01 ; * * * \mathrm{P} \leq 0.001$.

\subsection{Cancer cells with SUCNR1 knockdown show increased superoxide production}

As a result of oxidative phosphorylation, mitochondrial reactive oxygen species (mtROS) are produced by the ETC in the mitochondria [27]. Since knockdown of SUCNR1 caused an increase in mitochondrial OCR (Figure 3A), we next analyzed whether the elevated respiration affected mtROS production. For that reason, we transfected MKN-45, NCI-H460 and PSN-1 cells with siSUCNR1 or siNC and stained the cells with Hoechst 33342 (Hoechst) and 
MitoSOX is a membrane permeable probe, which is oxidized by superoxide but no other ROS species resulting in red fluorescence after binding to nucleic acids. The fluorescence analyses revealed a significant increase of mitochondrial superoxide in NCI-H460 and PSN-1 cells upon knockdown of SUCNR1 as well as incubation with Menadione (Figure 4A). No significant changes in MitoSOX fluorescence were detected in MKN-45 cells upon SUCNR1 knockdown or Menadione treatment suggesting that different ROS species might be produced in this cell line. Our data showing elevated superoxide levels together with the observed effects on OCR and ECAR in cancer cells with SUCNR1 knockdown implicate increased mitochondrial respiration as a cause for higher mtROS production in these cells.
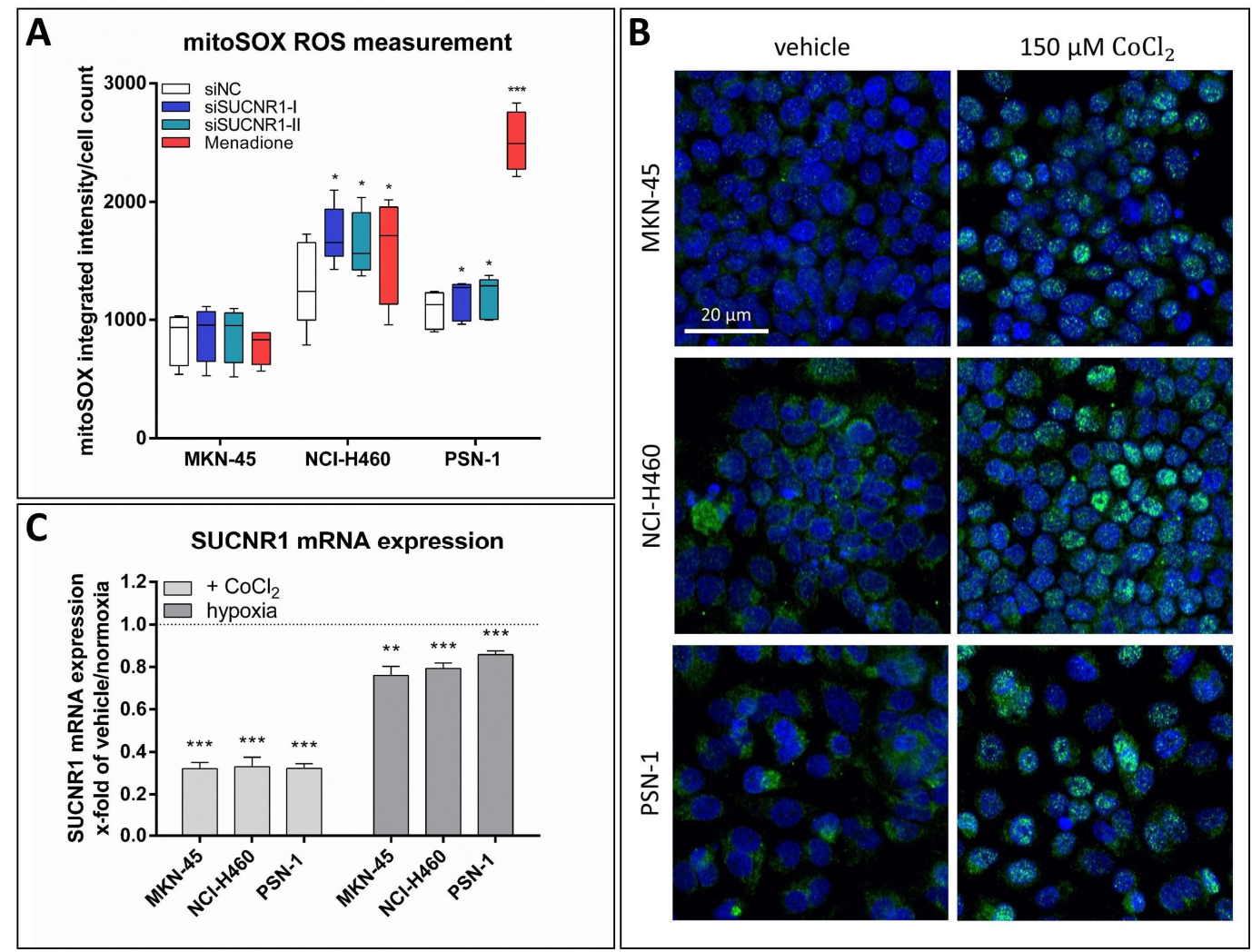

Figure 4: SUCNR1 knockdown increases mitochondrial ROS production.

A: Cancer cells were stained with Hoechst 33342 (Hoechst) and mitoSOX $48 \mathrm{~h}$ after transfection with siSUCNR1 or siNC to determine the production of mitochondrial superoxide. Images were automatically analyzed with the Celigo Image Cytometer (Nexcelom) and the integrated intensity of mitoSOX was normalized to the cell count (Hoechst-stained nuclei). Knockdown of SUCNR1 caused an increase of mitochondrial superoxide in NCI-H460 and PSN-1 cells. $40 \mu \mathrm{M}$ Menadione was used as a positive control. Data is shown as box and whiskers (min to $\max$ ) of $\mathrm{n}=5$ independent experiments. B, C: Cancer cells were incubated in HBSS + Glc + Gln in absence or presence of $150 \mu \mathrm{M} \mathrm{CoCl}_{2}$ or under hypoxia for $4 \mathrm{~h}$. B: Cancer cells were fixed, permeabilized, stained with primary mouse anti-HIF-1 $\alpha$ antibody and labelled with secondary AlexaFluor 488 goat anti-mouse antibody to confirm HIF-1 $\alpha$ activation. Nuclei were stained with Hoechst. Confocal imaging was performed with the CQ1 Confocal Imaging Cytometer (Yokogawa, Cenibra). C: SUCNR1 mRNA levels in cancer cell lines were determined by RT-qPCR showing a decrease induced by $\mathrm{CoCl}_{2}$ and hypoxia. Data is shown as mean $\pm \mathrm{SEM}$ of $\mathrm{n}=4$ independent experiments.

\subsection{Hypoxia causes a reduced SUCNR1 expression}

Since it is known that succinate levels are increased under hypoxic conditions, we analyzed whether hypoxia or treatment with hypoxia mimetic cobalt chloride $\left(\mathrm{CoCl}_{2}\right)$ [28] affects SUCNR1 mRNA expression. $\mathrm{CoCl}_{2}$ induces high expression of hypoxia-inducible factor-1 $\alpha$ (HIF-1 $\alpha$ ), a transcription factor that, under normoxic conditions, is ubiquitinylated and subsequently degraded in the proteasome while it is stabilized during hypoxia $[9,12]$. We 
immunocytochemical staining of the cancer cells in absence and presence of $\mathrm{CoCl}_{2}$ by confocal imaging (Figure 4B). SUCNR1 mRNA expression was decreased by about $20 \%$ upon incubation under hypoxia and $70 \%$ upon addition of $150 \mu \mathrm{M} \mathrm{CoCl}_{2}$ in comparison to cells incubated under normoxic conditions or without $\mathrm{CoCl}_{2}$ (Figure 4C). The reduction of SUCNR1 mRNA expression under hypoxia/hypoxia-mimicking conditions suggests a less pronounced role of SUCNR1 under hypoxia when respiration is hindered due to limited oxygen availability.

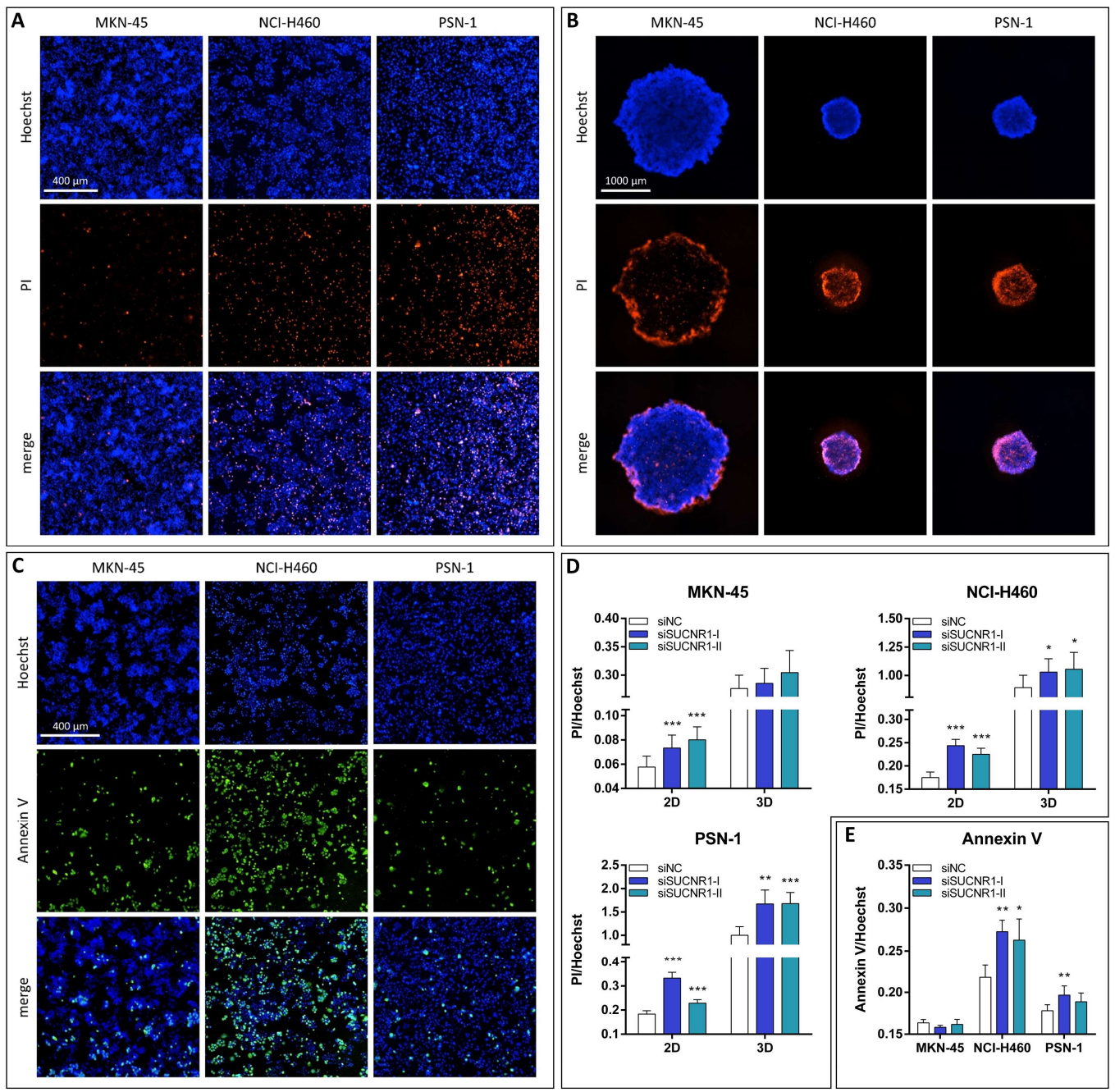

Figure 5: Knockdown of SUCNR1 decreases cancer cell survival.

A: 2D cultured cancer cells were transfected with SUCNR1-specific siRNA or siNC and stained with Hoechst 33342 (Hoechst, blue) and propidium iodide (PI, red) $72 \mathrm{~h}$ after transfection to determine total (Hoechst) and dead (PI) cell count. B: Cancer cells were seeded in ultra-low attachment plates to form 3D spheroids and transfected with siSUCNR1 or siNC. $72 \mathrm{~h}$ after transfection Hoechst and PI were added and blue/red fluorescence intensity was measured. C: 2D cultured cancer cells were transfected with SUCNR1-specific siRNA or siNC and stained with Hoechst (blue) and Annexin V-FITC (green) $24 \mathrm{~h}$ after transfection to determine total (Hoechst) and apoptotic (Annexin V) cell count. D: Knockdown of SUCNR1 in cancer cells caused an increase of dead cells in 2D and 3D cell cultures, which was determined with the Celigo Image Cytometer as cell count (2D) and fluorescence intensity (3D), respectively. E: Knockdown of SUCNR1 in cancer cells caused an increase of apoptotic cells, which was determined with the Celigo Image Cytometer as cell count. D, E: Data is shown as PI (D) or Annexin V (E) cell count (2D) or fluorescence intensity (3D) divided by Hoechst cell count (2D) or fluorescence intensity (3D) (mean \pm SEM) of $n \geq 4$ independent experiments each carried out in triplicates. Statistical analyses were performed using a repeated measures One-Way ANOVA. * $\mathrm{P} \leq 0.05 ; * * \mathrm{P} \leq 0.01 ; * * * \mathrm{P} \leq 0.001$.

\subsection{Knockdown of SUCNR1 affects survival of 2D and 3D cultured cancer cells}

Next, we analyzed the relevance of SUCNR1 for cancer cell survival. We transfected 2D and $3 \mathrm{D}$ cultured cancer cells with siSUCNR1-I or siSUCNR1-II in comparison to siNC. We used 
and cancer cell survival by imaging and automatic analysis. $72 \mathrm{~h}$ after transfection with siRNA, the cancer cells were stained with Hoechst (total cell count) and propidium iodide (PI, dead cell count) (Figure 5A, 5B). To further elucidate the effect of SUCNR1 knockdown on cancer cell death we stained the cells with Annexin V-AF488 (Annexin V) to determine the apoptotic cell count $24 \mathrm{~h}$ after siRNA transfection (Figure 5C). We found no differences in confluence or spheroid volume, respectively, when comparing siNC versus siSUCNR1 transfected cells (Figure S2C). The knockdown of SUCNR1 caused an increase of the PI/Hoechst ratio in MKN45 cells in 2D but not 3D cell culture (Figure 5D). Similarly, we found more dead cells in NCIH460 and PSN-1 cells after knockdown of SUCNR1, which resulted in an increased PI/Hoechst ratio in both 2D and 3D cell cultures (Figure 5D). The Annexin V/Hoechst staining showed an increase of apoptotic cells for the NCI-H460 and a similar trend for the PSN-1 but not MKN45 cells (Figure 5E).
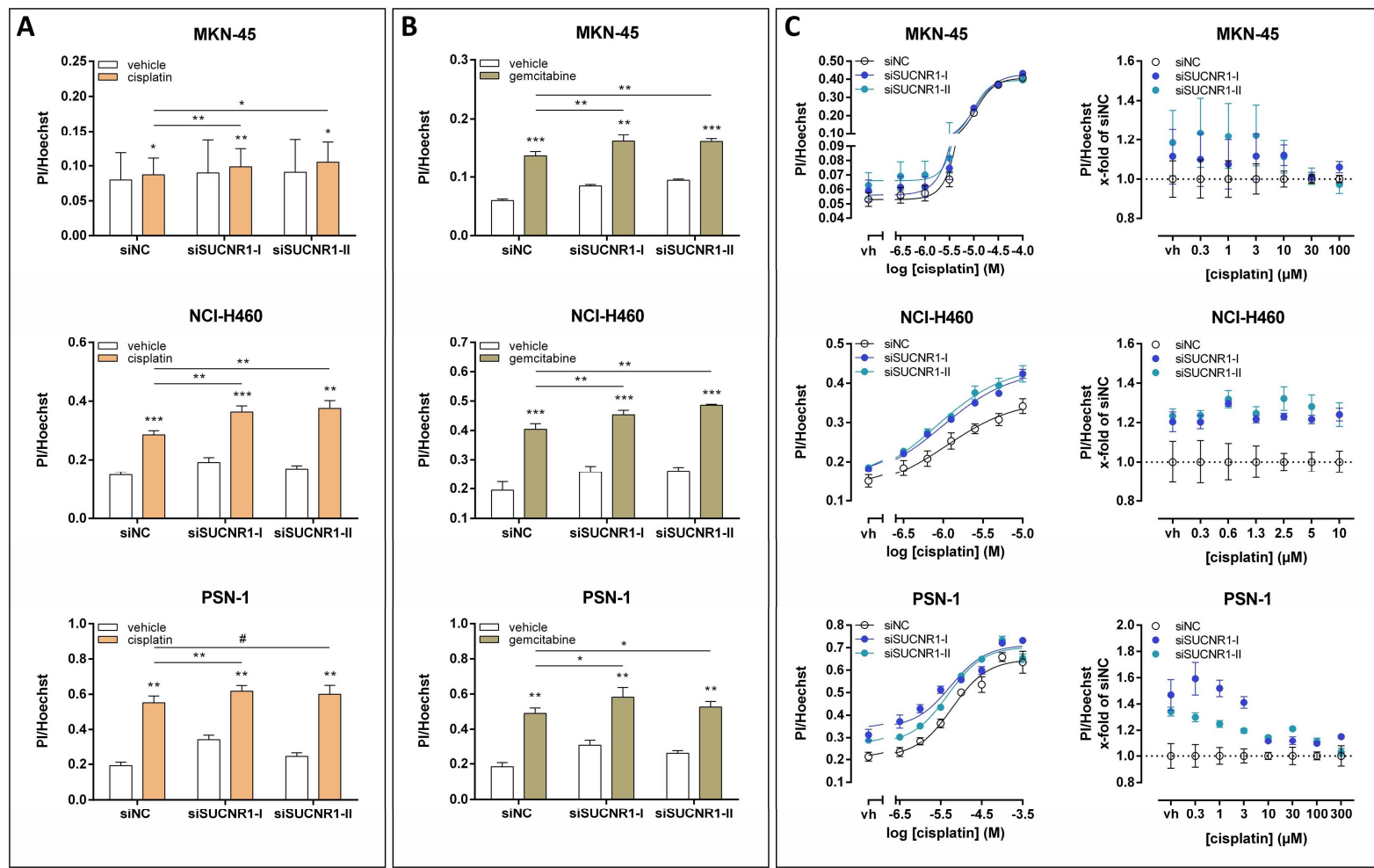

542 Figure 6: Knockdown of SUCNR1 in combination with cisplatin or gemcitabine further decreases cancer cell survival.

A, B: Cancer cells were transfected with siSUCNR1 or siNC and after $24 \mathrm{~h} 10 \mu \mathrm{M}$ cisplatin (A) or $10 \mu \mathrm{M}$ gemcitabine (B), respectively, was added to the medium. $72 \mathrm{~h}$ after siRNA transfection cells were stained with Hoechst 33342 (Hoechst, blue) and propidium iodide (PI, red) to determine total (Hoechst) and dead (PI) cell count with the Celigo Image Cytometer (Nexcelom). SUCNR1 knockdown and simultaneous treatment with the chemotherapeutics cisplatin and gemcitabine increased cancer cell death. Data is shown as PI cell count divided by Hoechst cell count (mean \pm SEM) of $n \geq 3$ independent experiments each carried out in triplicates. C: Cancer cells were transfected with siSUCNR1 or siNC and after $24 \mathrm{~h}$ different concentrations of cisplatin were added to the medium. $72 \mathrm{~h}$ after siRNA transfection cells were stained with Hoechst (blue) and PI (red) to determine total (Hoechst) and dead (PI) cell count with the Celigo Image Cytometer. SUCNR1 knockdown and simultaneous treatment with various concentrations of cisplatin (vh: vehicle) increased cancer cell death. Data is shown as PI cell count divided by Hoechst cell count (mean \pm SEM) of $n \geq 3$ independent experiments each carried out in triplicates. Statistical analyses were performed using a repeated measures One-Way ANOVA. ${ }^{*} \mathrm{P} \leq 0.1 ; * \mathrm{P} \leq$ $0.05 ; * * \mathrm{P} \leq 0.01 ; * * * \mathrm{P} \leq 0.001$.

\subsection{SUCNR1 knockdown in combination with cisplatin or gemcitabine treatment further} reduces cancer cell survival frequently used for treatment of solid tumors [29], and the nucleoside gemcitabine [30]. We 
combined knockdown of SUCNR1 in MKN-45, NCI-H460 and PSN-1 cells with cisplatin or gemcitabine treatment, respectively and compared the effect on cancer cell death to chemotherapeutic treatment alone. We found that the survival of cancer cells with reduced SUCNR1 expression was even further decreased in combination with cisplatin (Figure 6A) or gemcitabine (Figure 6B) treatment. In MKN-45 cells, the addition of cisplatin only slightly increased cancer cell death (Figure 6A). Nevertheless, in presence of cisplatin or gemcitabine cell death was increased when SUCNR1 was knocked down as compared to siNC. These results suggest that SUCNR1 might be a potential target for combination therapy with cisplatin or gemcitabine to treat cancer more effectively. To further elucidate the potential effect of combining cisplatin treatment with SUCNR1 knockdown, a concentration response curve of cisplatin in cells transfected with siNC versus siSUCNR1 was determined (Figure 6C). These analyses showed that, at various concentrations of cisplatin, cancer cells with SUCNR1 knockdown displayed an increased amount of dead cells in comparison to siNC transfected cells (Figure 6C). In MKN-45 cells, the rather minor effect observed was only present at lower concentrations of cisplatin (Figure 6C).

\section{Discussion}

In the present study, we link SUCNR1 to cellular metabolism in three cancer cell lines of different origin: the gastric adenocarcinoma cell line MKN-45, the lung cancer cell line NCI-H460 and the pancreatic adenocarcinoma cell line PSN-1. Various types of cancer exhibit increased SUCNR1 expression including human lung [11], ovarian [18], renal and pancreatic cancer [31]. Further, succinate itself is an increasingly recognized oncometabolite, known to drive cancer progression via inhibition of prolyl hydroxylase activity and subsequent activation of HIF-1 $\alpha[12,32]$ and to upregulate vascular endothelial growth factor expression via SUCNR1 [33].

Here, we confirmed functional presence of SUCNR1 in three cancer cell lines showing SUCNR1-mediated $\mathrm{G} \alpha_{\mathrm{i}}$, Akt and ERK 1/2 signaling but found no evidence for SUCNR1induced $\mathrm{Ga}_{\mathrm{q}} / \mathrm{Ca}^{2+}$ signaling (Figure 1B-F, S1A). In the presence of Gln, SUCNR1 mRNA levels were increased compared to Glc alone suggesting that the receptor may be involved in the regulation of cellular Gln metabolization (Figure 2A). Moreover, the increased OCR indicates that all three SUCNR1-expressing cancer cell lines are dependent on Gln for mitochondrial respiration (Figure 2C). Since the metabolic activity was tested in complete absence of Glc, the substrate for aerobic glycolysis and subsequent lactate production, the ECAR in presence of Gln alone is very low compared to Glc (Figure 2E) [23,34]. However, all three cancer cell lines, which highly express SUCNR1, exhibited only low metabolic activity in presence of Glc alone (Figure 2C, 6A). This hints to a link between addiction to Gln and SUCNR1 while an apparent connection between aerobic glycolysis, another prominent feature of cancer cell metabolism, and SUCNR1 is lacking. Another fundamental metabolic pathway, which is the main source of biosynthetic precursors but also energy is the TCA cycle, in which succinate is one of the central metabolites [7,8]. No link between TCA cycle throughput and SUCNR1 has so far been established. Here, we used addition of pyruvate as substrate to fuel the TCA cycle and itaconate, an inhibitor of SDH/complex II [25], which consequently downregulates TCA cycle throughput (Figure 2C-2H). In all three cell lines, significantly increased maximal respiration was found, when pyruvate was added as fuel of the TCA cycle while inhibition by itaconate had the opposite effect (Figure 2C, 2G). These results indicate that under conditions of high energy demand all three cell lines, metabolize Gln and pyruvate aerobically in the TCA cycle. For MKN-45 and NCI-H460 cells this was also true under basal conditions (Figure 2C). To provide further evidence for the link between TCA cycle throughput and SUCNR1, siRNA-mediated knockdown experiments were performed. These analyses revealed that both OCR and ECAR, in absence of Glc serving as indicator of elevated production of bicarbonate and protons during 
mitochondrial respiration, were increased in all three cancer cell lines with SUCNR1 as compared to negative control (Figure 3A, 3B, 6A).

612 These results, together with the up-regulated SUCNR1 expression and OCR in presence of Gln (Figure 2A, 2C), implicate that SUCNR1 is crucial for Gln-addicted cancer cells and involved in the limitation of TCA cycle throughput but also mitochondrial respiration (Figure 7A, 7B). Since the differences in metabolic phenotype comparing siNC with siSUCNR1 transfected cells were absent in presence of Gln and pyruvate in NCI-H460 and PSN-1 cells (Figure S2A, S2B), we conclude that in these cell lines SUCNR1 rather regulates Gln metabolism than the TCA cycle itself. High respiratory rates may be associated with an increased production of mitochondrial reactive oxygen species (mtROS) [35]. Here, a significant increase in mitochondrial superoxide upon knockdown of SUCNR1 in NCI-H460 and PSN-1 cells, but not MKN-45 cells was found (Figure 4A). Since in MKN-45 also no effect of Menadione on mitochondrial superoxide levels was observed, we conclude that other cellular ROS species like hydroxyl radicals or hydrogen peroxide, which were not detected in our analyses, may be increased when SUCNR1 is knocked down and/or OCR is increased.

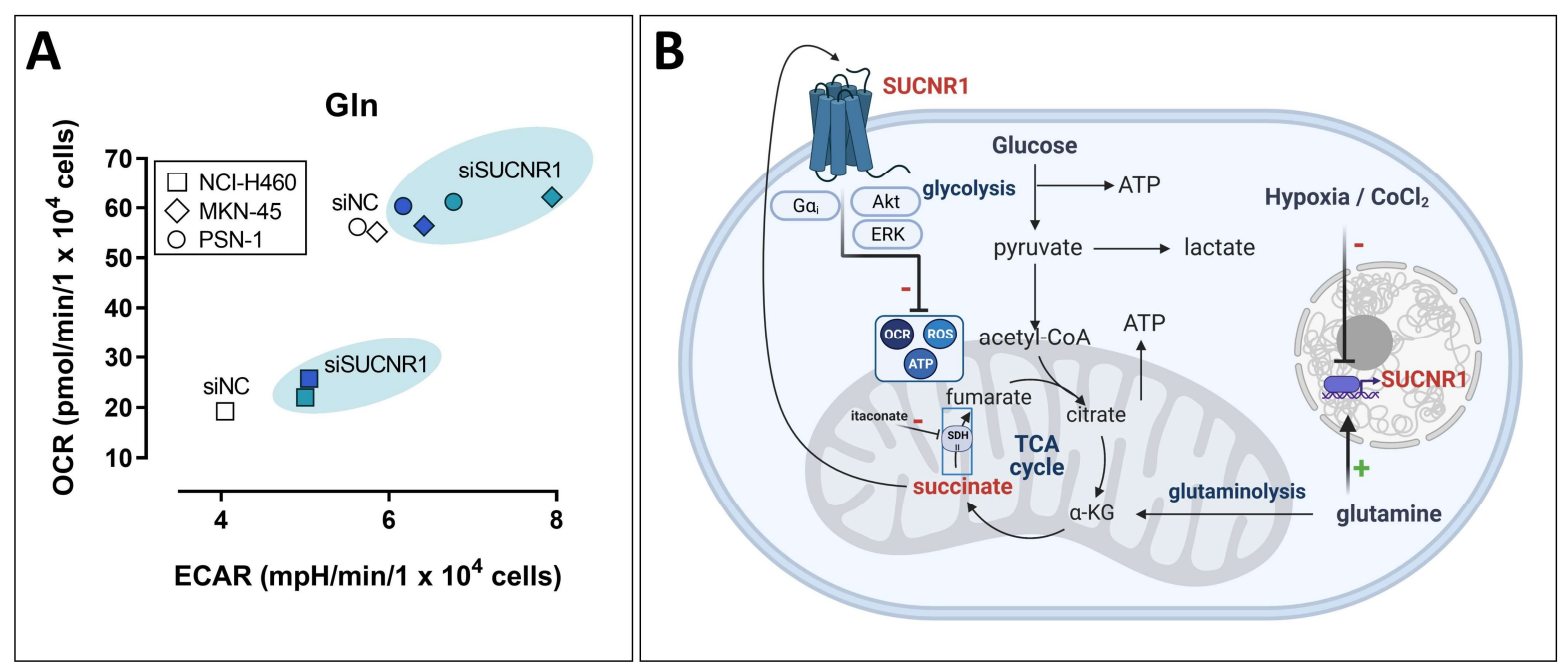

Figure 7: Metabolic phenotype of cancer cell lines and the link to SUCNR1.

A: Knockdown of SUCNR1 in presence of Gln caused a metabolic shift towards a more aerobic phenotype in all analyzed cancer cell lines. B: SUCNR1 regulates mitochondrial respiration, TCA cycle throughput and ROS production via a negative feedback mechanism. SUCNR1 mRNA levels are increased by Gln and decreased under hypoxia and in presence of the hypoxia mimetic $\mathrm{CoCl}_{2}$.

Future analyses will have to focus on establishing the link between SUCNR 1-induced $\mathrm{G} \alpha_{\mathrm{i}}$, ERK and Akt signaling and the diverse known regulatory mechanisms of TCA cycle throughput that result in the observed changes of metabolic phenotype. These potential regulatory points include the regulation of the pyruvate dehydrogenase complex by PDKs [36], induction of TRAP1 which acts as an inhibitor of SDH/complex II [37] or the regulation of Glutaminase -1 by c-Myc or HIF-1 $\alpha$ (reviewed in $[38,39]$ ). A previous study suggested the transcription factor CCAAT enhancer binding protein $\beta$ (C/EBP $\beta$ ), as downstream target of SUCNR1 activation [40]. Important regulatory roles in cellular processes including proliferation, transformation, metabolism, survival and/or apoptosis are known for C/EBP $\beta$ (reviewed in[41]).

Another aspect we addressed here is whether the knockdown of SUCNR1 accompanied by the metabolic effects of increased OCR and mtROS affects the survival of the analyzed cancer cell lines. Many cancers are treated with platinum-based drugs like the chemotherapeutic agent cisplatin $[42,43]$ or the nucleoside gemcitabine [30]. Since cisplatin causes toxic side effects, combination therapies with other drugs are applied [42]. In our study, we found that the combination of siSUCNR1 transfection and cisplatin or gemcitabine treatment reduced cancer cell survival (Figure 6A, 6B). Thus, antagonist-mediated SUCNR1 inhibition may render lower concentrations of cisplatin necessary, thereby reducing its toxic side effects while achieving the 
same therapeutic effects as shown in the cisplatin dose-response curves (Figure 6C). Such specific SUCNR1 antagonists are already available for research (MedChemExpress: NF-56EJ40) and novel SUCNR1 inhibitors with improved oral exposure are currently being developed [44]. Thus, we suggest that SUCNR1 represents a potential pharmacological target for cancer treatment.

In summary, our data demonstrates that SUCNR1 signaling through activation of $G \alpha_{i}$, ERK and Akt constitutes a negative feedback mechanism in Gln-addicted cancer cells, limiting TCA cycle throughput, mitochondrial respiration and ROS production in presence of oxygen. Under hypoxia, SUCNR1 expression is decreased. These SUCNR1-mediated metabolic regulatory mechanisms are relevant for cancer cell proliferation and may be therapeutically exploited in the future.

\section{Acknowledgements}

We thank Christoph Enz for the possibility to use the CQ1 and Michael Schell for the confocal imaging support. Further, we thank Ulf Wagner and Kathleen Friedrich for providing and assisting with the hypoxic chamber.

\section{Funding}

This work was supported by the German Research Foundation (www.dfg.de): CS (STA 1265/3-1) and by the Medical Faculty, University Leipzig (https://www.uniklinikumleipzig.de/wissenschaft-forschung/forschungs-administration/forschungsförderung).

\section{Author contributions}

All authors acquired data and carried out experiments. PR, ADL, PK, RK and CS analyzed and interpreted data. PR and CS visualized the data. PR drafted the manuscript. CS conceptually and experimentally designed the work, supervised the study and wrote the manuscript. All authors discussed the results and implications and commented on the manuscript at all stages. All authors read and approved the final manuscript.

\section{Conflict of interest statement}

The authors have declared that no competing interests exist.

\section{References}

[1] H.A. Coller, Is Cancer a Metabolic Disease?, Am. J. Pathol. 184 (2014) 4-17. https://doi.org/10.1016/j.ajpath.2013.07.035.

[2] D. Hanahan, R.A. Weinberg, Hallmarks of Cancer: The Next Generation, Cell 144 (2011) 646-674. https://doi.org/10.1016/j.cell.2011.02.013.

[3] O. Warburg, On respiratory impairment in cancer cells, Science (N.Y.) 124 (1956).

[4] P.P. Hsu, D.M. Sabatini, Cancer Cell Metabolism: Warburg and Beyond, Cell 134 (2008) 703-707. https://doi.org/10.1016/j.cell.2008.08.021.

[5] R.J. DeBerardinis, A. Mancuso, E. Daikhin, I. Nissim, M. Yudkoff, S. Wehrli, C.B. Thompson, Beyond aerobic glycolysis: Transformed cells can engage in glutamine metabolism that exceeds the requirement for protein and nucleotide synthesis, PNAS 104 (2007) 19345-19350. https://doi.org/10.1073/pnas.0709747104.

[6] D.R. Wise, C.B. Thompson, Glutamine Addiction: A New Therapeutic Target in Cancer, Trends Biochem. Sci. 35 (2010) 427-433. https://doi.org/10.1016/j.tibs.2010.05.003.

[7] A.C.B. Sant'Anna-Silva, J.A. Perez-Valencia, M. Sciacovelli, C. Lalou, S. Sarlak, L. Tronci, E. Nikitopoulou, A.T. Meszaros, C. Frezza, R. Rossignol, E. Gnaiger, H. Klocker, Succinate Anaplerosis Has an Onco-Driving Potential in Prostate Cancer Cells, Cancers (Basel) 13 (2021). https://doi.org/10.3390/cancers13071727. 
[8] I. Martínez-Reyes, N.S. Chandel, Mitochondrial TCA cycle metabolites control physiology and disease, Nat. Commun. 11 (2020) 1-11. https://doi.org/10.1038/s41467019-13668-3.

[9] T. Zhao, X. Mu, Q. You, Succinate: An initiator in tumorigenesis and progression, Oncotarget 8 (2017) 53819-53828. https://doi.org/10.18632/oncotarget.17734.

[10] P. Xekouki, K. Pacak, M. Almeida, C.A. Wassif, P. Rustin, M. Nesterova, de la Luz Sierra, M., J. Matro, E. Ball, M. Azevedo, A. Horvath, C. Lyssikatos, M. Quezado, N. Patronas, B. Ferrando, B. Pasini, A. Lytras, G. Tolis, C.A. Stratakis, Succinate Dehydrogenase (SDH) D Subunit (SDHD) Inactivation in a Growth-Hormone-Producing Pituitary Tumor: A New Association for SDH?, J. Clin. Endocrinol. Metab. 97 (2012) E357-66. https://doi.org/10.1210/jc.2011-1179.

[11] J.-Y. Wu, T.-W. Huang, Y.-T. Hsieh, Y.-F. Wang, C.-C. Yen, G.-L. Lee, C.-C. Yeh, Y.J. Peng, Y.-Y. Kuo, H.-T. Wen, H.-C. Lin, C.-W. Hsiao, K.K. Wu, H.-J. Kung, Y.-J. Hsu, C.-C. Kuo, Cancer-Derived Succinate Promotes Macrophage Polarization and Cancer Metastasis via Succinate Receptor, Mol. Cell 77 (2020) 213-227.e5. https://doi.org/10.1016/j.molcel.2019.10.023.

[12] M.A. Selak, S.M. Armour, E.D. MacKenzie, H. Boulahbel, D.G. Watson, K.D. Mansfield, Y. Pan, M.C. Simon, C.B. Thompson, E. Gottlieb, Succinate links TCA cycle dysfunction to oncogenesis by inhibiting HIF-alpha prolyl hydroxylase, Cancer cell 7 (2005). https://doi.org/10.1016/j.ccr.2004.11.022.

[13] A. Nagao, M. Kobayashi, S. Koyasu, C.C.T. Chow, H. Harada, HIF-1-Dependent Reprogramming of Glucose Metabolic Pathway of Cancer Cells and Its Therapeutic Significance, Int. J. Mol. Sci. 20 (2019). https://doi.org/10.3390/ijms20020238.

[14] J. Gilissen, F. Jouret, B. Pirotte, J. Hanson, Insight into SUCNR1 (GPR91) structure and function, Pharmacol. Ther. 159 (2016) 56-65. https://doi.org/10.1016/j.pharmthera.2016.01.008.

[15] C. Högberg, O. Gidlöf, C. Tan, S. Svensson, J. Nilsson-Öhman, D. Erlinge, B. Olde, Succinate independently stimulates full platelet activation via cAMP and phosphoinositide 3-kinase- $\beta$ signaling, J. Thromb. Haemost. 9 (2011). https://doi.org/10.1111/j.1538-7836.2010.04158.x.

[16] J.H. Robben, R.A. Fenton, S.L. Vargas, H. Schweer, J. Peti-Peterdi, P.M.T. Deen, G. Milligan, Localization of the succinate receptor in the distal nephron and its signaling in polarized MDCK cells, Kidney International 76 (2009) 1258-1267. https://doi.org/10.1038/ki.2009.360.

[17] J. Cosín-Roger, D. Ortiz-Masia, M.D. Barrachina, S. Calatayud, Metabolite Sensing GPCRs: Promising Therapeutic Targets for Cancer Treatment?, Cells 9 (2020). https://doi.org/10.3390/cells9112345.

[18] J. Zhang, Q. Zhang, Y. Yang, Q. Wang, Association Between Succinate Receptor SUCNR1 Expression and Immune Infiltrates in Ovarian Cancer, Front. Mol. Biosci. 7 (2020). https://doi.org/10.3389/fmolb.2020.00150.

[19] D.M. Matlac, K. Hadrava Vanova, N. Bechmann, S. Richter, J. Folberth, H.K. Ghayee, G.-B. Ge, L. Abunimer, R. Wesley, R. Aherrahrou, M. Dona, Á.M. Martínez-Montes, B. Calsina, M.J. Merino, M. Schwaninger, P.M.T. Deen, Z. Zhuang, J. Neuzil, K. Pacak, H. Lehnert, S.M.J. Fliedner, Succinate Mediates Tumorigenic Effects via Succinate Receptor 1: Potential for New Targeted Treatment Strategies in Succinate Dehydrogenase Deficient Paragangliomas, Front. Endocrinol. (Lausanne) 12 (2021) 589451. https://doi.org/10.3389/fendo.2021.589451.

[20] C. Stäubert, J. Bohnekamp, T. Schöneberg, Determinants involved in subtype-specific functions of rat trace amine-associated receptors 1 and 4, Br. J. Pharmacol. 168 (2013) 1266-1278. https://doi.org/10.1111/bph.12020. 
[21] P. Geubelle, J. Gilissen, S. Dilly, L. Poma, N. Dupuis, C. Laschet, D. Abboud, A. Inoue, F. Jouret, B. Pirotte, J. Hanson, Identification and pharmacological characterization of succinate receptor agonists, Br. J. Pharmacol. 174 (2017) 796-808. https://doi.org/10.1111/bph.13738.

[22] Z.-G. Gao, K.A. Jacobson, On the Selectivity of the Gaq Inhibitor UBO-QIC: A Comparison with the Gai Inhibitor Pertussis Toxin, Biochem. Pharmacol. 107 (2016) 59-66. https://doi.org/10.1016/j.bcp.2016.03.003.

[23] L.S. Pike Winer, M. Wu, Rapid Analysis of Glycolytic and Oxidative Substrate Flux of Cancer Cells in a Microplate, PLoS ONE 9 (2014). https://doi.org/10.1371/journal.pone.0109916.

[24] J.R. Casey, S. Grinstein, J. Orlowski, Sensors and regulators of intracellular pH, Nat. Rev. Mol. Cell Biol. 11 (2010) 50-61. https://doi.org/10.1038/nrm2820.

[25] T. Cordes, A. Lucas, A.S. Divakaruni, A.N. Murphy, P. Cabrales, C.M. Metallo, Itaconate modulates tricarboxylic acid and redox metabolism to mitigate reperfusion injury, Mol. Metab. 32 (2020) 122-135. https://doi.org/10.1016/j.molmet.2019.11.019.

[26] L. Tretter, A. Patocs, C. Chinopoulos, Succinate, an intermediate in metabolism, signal transduction, ROS, hypoxia, and tumorigenesis, Biochim. Biophys. Acta 1857 (2016) 1086-1101. https://doi.org/10.1016/j.bbabio.2016.03.012.

[27] F. Scialò, D.J. Fernández-Ayala, A. Sanz, Role of Mitochondrial Reverse Electron Transport in ROS Signaling: Potential Roles in Health and Disease, Front. Physiol. 8 (2017) 428. https://doi.org/10.3389/fphys.2017.00428.

[28] J. Muñoz-Sánchez, M.E. Chánez-Cárdenas, The use of cobalt chloride as a chemical hypoxia model, J. Appl. Toxicol. 39 (2019) 556-570. https://doi.org/10.1002/jat.3749.

[29] M. Kleih, K. Böpple, M. Dong, A. Gaißler, S. Heine, M.A. Olayioye, W.E. Aulitzky, F. Essmann, Direct impact of cisplatin on mitochondria induces ROS production that dictates cell fate of ovarian cancer cells, Cell Death Dis. 10 (2019) 1-12. https://doi.org/10.1038/s41419-019-2081-4.

[30] D.S. Gesto, N.M. Cerqueira, P.A. Fernandes, M.J. Ramos, Gemcitabine: a critical nucleoside for cancer therapy, Current medicinal chemistry 19 (2012). https://doi.org/10.2174/092986712799320682.

[31] X. Terra, V. Ceperuelo-Mallafré, C. Merma, E. Benaiges, R. Bosch, P. Castillo, J.C. Flores, X. León, I. Valduvieco, N. Basté, M. Cámara, M. Lejeune, J. Gumà, J. Vendrell, I. Vilaseca, S. Fernández-Veledo, F.X. Avilés-Jurado, Succinate Pathway in Head and Neck Squamous Cell Carcinoma: Potential as a Diagnostic and Prognostic Marker, Cancers (Basel) 13 (2021) 1653. https://doi.org/10.3390/cancers13071653.

[32] P. Koivunen, M. Hirsilä, A.M. Remes, I.E. Hassinen, K.I. Kivirikko, J. Myllyharju, Inhibition of hypoxia-inducible factor (HIF) hydroxylases by citric acid cycle intermediates: possible links between cell metabolism and stabilization of HIF, J. Biol. Chem. 282 (2007). https://doi.org/10.1074/jbc.M610415200.

[33] X. Mu, T. Zhao, C. Xu, W. Shi, B. Geng, J. Shen, C. Zhang, J. Pan, J. Yang, S. Hu, Y. Lv, H. Wen, Q. You, Oncometabolite succinate promotes angiogenesis by upregulating VEGF expression through GPR91-mediated STAT3 and ERK activation, Oncotarget 8 (2017) 13174-13185. https://doi.org/10.18632/oncotarget.14485.

[34] M. Wu, A. Neilson, A.L. Swift, R. Moran, J. Tamagnine, D. Parslow, S. Armistead, K. Lemire, J. Orrell, J. Teich, S. Chomicz, D.A. Ferrick, Multiparameter metabolic analysis reveals a close link between attenuated mitochondrial bioenergetic function and enhanced glycolysis dependency in human tumor cells, Am. J. Physiol. 292 (2007). https://doi.org/10.1152/ajpcell.00247.2006.

[35] M.P. Murphy, How mitochondria produce reactive oxygen species, Biochem. J. 417 (2009) 1-13. https://doi.org/10.1042/BJ20081386. 
[36] B.L. Woolbright, G. Rajendran, R.A. Harris, J.A. Taylor, Metabolic Flexibility in Cancer: Targeting the Pyruvate Dehydrogenase Kinase:Pyruvate Dehydrogenase Axis, Mol. Cancer Ther. 18 (2019) 1673-1681. https://doi.org/10.1158/1535-7163.MCT-190079.

[37] M. Sciacovelli, G. Guzzo, V. Morello, C. Frezza, L. Zheng, N. Nannini, F. Calabrese, G. Laudiero, F. Esposito, M. Landriscina, P. Defilippi, P. Bernardi, A. Rasola, The Mitochondrial Chaperone TRAP1 Promotes Neoplastic Growth by Inhibiting Succinate Dehydrogenase, Cell Metab. 17 (2013) 988-999. https://doi.org/10.1016/j.cmet.2013.04.019.

[38] W.P. Katt, R.A. Cerione, Glutaminase regulation in cancer cells: a druggable chain of events, Drug Discov. Today 19 (2014) 450-457. https://doi.org/10.1016/j.drudis.2013.10.008.

[39] Z. Wang, F. Liu, N. Fan, C. Zhou, D. Li, T. Macvicar, Q. Dong, C.J. Bruns, Y. Zhao, Targeting Glutaminolysis: New Perspectives to Understand Cancer Development and Novel Strategies for Potential Target Therapies, Front. Oncol. 0 (2020) 2321. https://doi.org/10.3389/fonc.2020.589508.

[40] T. Li, J. Hu, F. Gao, X. Du, Y. Chen, Q. Wu, Transcription factors regulate GPR91mediated expression of VEGF in hypoxia-induced retinopathy, Sci. Rep. 7 (2017) 45807. https://doi.org/10.1038/srep45807.

[41] M. Tolomeo, S. Grimaudo, The "Janus" Role of C/EBPs Family Members in Cancer Progression, Int. J. Mol. Sci. 21 (2020) 4308. https://doi.org/10.3390/ijms21124308.

[42] Q. Zhang, Q.-B. Lu, New combination chemotherapy of cisplatin with an electrondonating compound for treatment of multiple cancers, Sci. Rep. 11 (2021) 1-13. https://doi.org/10.1038/s41598-020-80876-z.

[43] J.T. Hartmann, H.P. Lipp, Toxicity of platinum compounds, Expert Opin. Pharmacoth. 4 (2003). https://doi.org/10.1517/14656566.4.6.889.

[44] J. Velcicky, R. Wilcken, S. Cotesta, P. Janser, A. Schlapbach, T. Wagner, P. Piechon, F. Villard, R. Bouhelal, F. Piller, S. Harlfinger, R. Stringer, D. Fehlmann, K. Kaupmann, A. Littlewood-Evans, M. Haffke, N. Gommermann, Discovery and Optimization of Novel SUCNR1 Inhibitors: Design of Zwitterionic Derivatives with a Salt Bridge for the Improvement of Oral Exposure, J. Med. Chem. 63 (2020) 9856-9875. https://doi.org/10.1021/acs.jmedchem.0c01020. 